\title{
Management strategy of Corona Virus Disease- 2019 in quarantine zones outside hospitals: Analysis of 1232 cases from a district in Wuhan
}

\section{Xiaojian Zhu}

Tongji Hospital of Tongji Medical College of Huazhong University of Science and Technology Jue Wang

Tongji Hospital of Tongji Medical College of Huazhong University of Science and Technology Department of Radiology

Hao Wang

The Dean of Hanzhengjie Community Healthy center of Qiaokou district

\section{Yutong Tang}

Wuhan union hospital

Shu Zhou

Wuhan union hospital

Yuxiao Zhang

Wuhan university of health science

\section{Lianzhen Wang}

Wuhan No.1 hospital

\section{Shengxin Liu}

The third people's hospital of Hubei Province

\section{Yanmin Zhang}

Wuhan No.1 hospital

\section{Wen Liu}

Wuhan union hospital

\section{Wenlan Fu}

The third people's hospital of hubei province

\section{Yuanping Cai}

Wuhan fourth hospital

\section{Yu Tao}

Tongji Hospital of Tongji Medical College of Huazhong University of Science and Technology

\section{Fang Liu}

The third people' s hospital of hubei province

Jun Zhou ( $\sim$ junzhouhbzsyy@163.com )

The third people' hospital of Hubei Province 
Research

Keywords: Corona Virus Disease 2019, Novel coronavirus pneumonia, quarantine zones

Posted Date: May 18th, 2020

DOI: https://doi.org/10.21203/rs.3.rs-28749/v1

License: (c) (i) This work is licensed under a Creative Commons Attribution 4.0 International License. Read Full License 


\section{Abstract}

The Corona Virus Disease 2019 (COVID-19) has evolved into a global pandemic in the early 2020. Management strategy outside hospitals of the suspected cases, close contacts and discharged patients might be as important as treatment in hospital. We analyzed information from 1232 cases at 14 hotels (requisitioned as quarantine zones) in Qiaokou district, Wuhan during Feb 8th to Mar 4th 2020. Abide by the unquarantine and hospitalization standard, 603 (48.94\%) cases were released from quarantine zones; $540(43.83 \%)$ cases were sent to hospital for further medical care. $89(7.22 \%)$ cases remained on quarantine up to the end of the analysis. The reasons for cases sent to the hospital for treatment were either positive for COVID-19 nucleic acid test, progression in pulmonary CT scan, or aggravation of symptoms. 11/59 patients switched from negative to positive for nucleic acid test during stayed in quarantine zones after being discharged from the hospitals. In total, hospitalization and positive rate for COVID-19 nucleic acid test both decreased over time. The quarantine measures were important and played a pivotal role in identification of cardinal number, cutting off the transmission, reducing the scope of prevention and rehabilitation therapy. This protocol adopted in Wuhan provided countries worldwide with valuable experience.

\section{Background}

Corona Virus Disease 2019 (COVID-19) refers to pneumonia resulted from a novel coronavirus infection in $2019^{1}$. According to the Chinese Center for Disease Control and Prevention of Viral Diseases, the virus belongs to the $\beta$-coronavirus cluster, which comprised of a single-stranded ribonucleic acid (RNA) structure, with the possibility for rapid mutation ${ }^{2}$. Since then, the COVID-19 has evolved into a global pandemic in the early 2020; up to March $18^{\text {th }}$, there were more than 200,000 confirmed cases and the estimated number of individuals call for prevention and control of infection has reached billions. Based on epidemiological investigations, the incubation period of the virus is generally from 1 to 14 days in which the disease can be contagious, and the majority of the population is susceptible to the virus ${ }^{3}$. Unfortunately, there is no validated curative therapy such as vaccine or specific drug against COVID-19 so far. As a result, screening, isolation, symptomatic and supportive care are the major treatment procedures $^{1,2}$. In order to screen as many people as possible and maximumly cut off the transmission, Wuhan government began to designate hotels as quarantine zones from $1^{\text {th }}$ Feb, and equip them with basic medical care and testing abilities, in hope to properly screen suspected cases, close contacts with confirmed cases and to monitor discharged patients. This work briefly summarized the management process and clinical outcomes at 14 quarantine zones in the Qiaokou District of Wuhan from Feb $8^{\text {th }}$ to Mar $4^{\text {th }} 2020$.

\section{Methods}

Inclusion criteria and classification 
To clarify the effect and management process of quarantine zones on control of novel coronary pneumonia, we gathered information of all cases came to 14 hotels in Qiaokou district, Wuhan P. R. China from Feb $8^{\text {th }}$ to Mar $4^{\text {th }}, 2020$. The research protocol was assessed and approved by the Ethical Committee of the Tongji Hospital, Tongji medical college, Huazhong University of Science and Technology. For data analysis, 416 cases with incomplete information were excluded.

According to the characteristics and reasons for which they came to the quarantine zones, we divided the personnel into four groups: CT suspected, symptomatic suspected, close contacts and discharged patients. CT suspected cases were those who came to the quarantine zone because of imaging features of viral pneumonia, but with serum antibody ( $\operatorname{lgM}$ or $\lg G$ ) negative and nucleic acid test negative, symptomatic or asymptomatic. People with fever or respiratory symptoms were termed as symptomatic suspected cases (serum antibody (IgM or $\operatorname{lgG}$ ) negative and nucleic acid test negative, without viral pneumonia changes in CT scan when they came in). Close contacts: those who have history of close contact with confirmed cases in the absence of effective protection. Discharged patients: patients discharged from the designated hospital, whose CT scan showing significant improvement after treatment and nucleic acid test negative (2 consecutive times, more than one day apart from each other), and with improvement of general condition.

\section{Exclusion criteria}

People with the following characteristics could not stay at the quarantine zones: (1)nucleic acid test of COVID-19 positive at any time; (2)patients with serious complications/comorbidities such as metabolic diseases, cardiovascular and cerebrovascular diseases; (3)people who are unable to take care of themselves;(4) people who cannot guarantee completion of treatment plan and follow-up observations; (5) patients taking part in clinical trials.

\section{Management}

Every quarantine zone was attended by doctors and nurses to monitor body temperature, blood oxygen saturation, clinical symptoms at least once a day. During the isolation period, all cases could receive basic oral medications if needed. Nasopharyngeal swab samples were collected in each zone and sent to the CDC for COVID-19 nucleic acid tests. Most of the CT scan were performed and analyzed in the third people's hospital of HuBei Province. Nasopharyngeal swab and CT scan were given to each case once they came in, and at least one more time before they left the quarantine zones.

\section{Unquarantine standard}

All following four conditions must be met for de-isolation from quarantine zones: (1)cases should be in good physical status, with ECOG score 0-2 points, and without respiratory symptoms; (2) the latest CT scan suggested that the conditions of viral pneumonia significantly improved; (3)two consecutive nucleic acid tests were negative (more than one day apart from each other); (4) cases should have stayed for at least 14 days. 


\section{Hospitalization standard}

Cases should be transferred to the mobile cabin or designated hospitals for treatment as soon as possible if any one of following conditions were met: (1)COVID-19 nucleic acid positive at any time; (2) respiratory symptoms were aggravated and required for further medication; (3) CT scan indicating a unfavorable or worsening inflammatory changes in pulmonary system;(4)cases with worsened health condition during isolation, whether it's related or unrelated to viral pneumonia.

\section{Statistical Analysis}

Data collection was performed in accordance with the RECORD standard. The rate of different time points in the same group were evaluated by chi-square tests. All analyses were conducted with SPSS10.0 and $\mathrm{P}<0.05$ was considered as statistical significance.

\section{Patient and Public Involvement}

Patients were not involved in development of the research question and outcome measures, study design or conduct of this study.

\section{Results}

\section{Demographic and clinical characteristics}

We enrolled 1648 cases at 14 quarantine zones in Qiaokou district from Feb $8^{\text {th }}$ to Mar $4^{\text {th }} 2020$. We delineated 1,232 cases for further analysis and 416 cases with incomplete information were excluded. Among them, the median age was 52.00 years (2-93y), and the average age was 50.95y (2y-93y); M: $F=577: 655$. Based on the source of the population and the reasons for which those cases entered the quarantine zones, we artificially divided all cases into 4 groups (Table 1). There were 607 CT suspected cases $(49.27 \%)$, which refers to people with viral pneumonia changes in CT scan, having symptoms or not. Among them, M: $\mathrm{F}=271: 336$, and the median age was 53.00y (3-90y). Symptomatic suspected cases were count to 200 (16.24\%), who developed fever or respiratory symptoms but without classical CT changes when they came to the zones. Among them, M: F=97:103, and the median age was 51.0 (2-82y). In symptomatic group, fever is the most common symptom, followed by cough, dyspnea, chest congestion and asthma, etc. Another 366 (29.71\%) people who have histories of close contact with confirmed COVID-19 cases in absence of effective protections were termed as close contact cases. The $\mathrm{M}: \mathrm{F}=180: 186$ and median age was 51.00y (5-93y). Finally, in 59 (4.79\%) discharged patients, who had been confirmed COVID-19 positive, accepted treatment in designated hospitals and were asked to stay at the quarantine zones for at least 14 days before going back home, the $M: F=29: 30$ and the median age was $55.0(9-89 y)$.

\section{Clinical outcomes of the cases}


All cases were asked to stay at the quarantine zones for at least 14 days and allowed to go home when the criteria were met after the isolation time (Figure 1). Among all the 1232 cases, 603 (48.94\%) cases were successfully de-isolated from quarantine zones, 540 (43.83\%) cases were sent to hospitals for further medical care. As for the other remaining 89 (7.22\%) cases, who did not meet the standards of either de-isolation or hospitalization, were continued to be kept on medical observations at quarantine zones. For the subgroup analysis, 306/607 (50.41\%) CT suspected cases were hospitalized, 272 (44.81\%) terminated the isolation and $29(4.78 \%)$ cases remained in the quarantine zones. In symptomatic suspected group, 53/200 (26.50\%) cases needed further medical care in hospital; 114/200 (57.00\%) cases were released from quarantine and $33(16.50 \%)$ cases remained in isolation. For the close contacts, 164/366 (44.81\%) were hospitalized; 190 (51.91\%) went back home and 12 (3.28\%) stayed in quarantine. In 59 discharged patients from hospitals, 17 (28.81\%) patients needed to be re-hospitalized; 27 (45.76\%) patients went home from quarantine and $15(25.43 \%)$ patients remained in medical observation. The CT suspected group demonstrated the highest rate of hospitalization; Significant difference was found between CT suspected cases and symptomatic suspected cases $(50.41 \%$ vs $26.50 \%$, Chi-square value $=32.57, \mathrm{P}<0.05)$. The close contacts group displayed a similar hospitalization rate as in $\mathrm{CT}$ suspected group $(50.41 \%$ vs $44.81 \%, P>0.05)$. The hospitalization rate of female was lower than that of male $(244 / 655,37.25 \%$ vs $296 / 577,51.30 \%$, Chi-square value $=24.59, P<0.05)$. In terms of the age distribution, among the sample population, cases within $41-70 \mathrm{y}$ in age accounted for $63.04 \%$ of all the population, which was the main component of isolation and hospitalized.

\section{Reasons for hospitalization}

The main reasons for 540 cases who were sent to the hospital for further treatment were as follows (Table 2): 200/540 (37.04\%) were nucleic acid positive; 124 (22.96\%) were for progression in CT scan. While CT scan of all de-isolated cases revealed that the inflammation could be significantly absorbed than before, these 124 cases exacerbated from pulmonary infection, with mainly enlarged lesions or new lesions. 107 (19.81\%) cases were hospitalized because of new respiratory symptoms or with previous symptoms exacerbated, which mainly manifested as cough, fever, chest tightness etc. Besides, 109 $(20.19 \%)$ cases were for other reasons, including being unable to adapt to the life in isolation, need for treatment of comorbidities, etc.

For subgroup analysis (Table 2), 109/306 (35.62\%) CT suspected cases were positive for nucleic acid test; $94(30.72 \%)$ cases showed insignificant improvement or exacerbation in CT scan; $50(16.34 \%)$ cases were for aggravation of symptoms, and $53(17.32 \%)$ cases for other causes. For the symptomatic suspected cases, 21/53 (39.62\%) were positive of nucleic acid, 5 (9.43\%) suffered progression in CT, 18 (33.96\%) for aggravation of symptoms and 9 (16.98\%) for other reasons. In close contacts group, there were 59/164 (35.98\%) cases of nucleic acid positive, 22 (13.41\%) cases with changes of viral pneumonia or advance of the original lesions on CT scan, 38 (23.17\%) cases admitted to hospitals for symptoms, and $45(27.44 \%)$ cases admitted for other reasons. There were 11/17 (64.71\%) discharged patients who were detected positive for nucleic acid again, $3(17.65 \%)$ patients had progression in CT scan, 1 (5.88\%) patient had recurrence of symptoms, and $2(11.76 \%)$ patients were hospitalized for other reasons. 
Of all the reasons of hospitalization, one of the most essential is nucleic acid positive for COVID-19, which confirms a case as a patient. In total, 200/1232 (16.24\%) cases were detected as novel coronavirus positive. Among them, 109/200 (54.50\%) were from the CT suspected group, 21 (10.50\%) from the symptomatic suspected group, 59 (29.5\%) from the close contacts group, and 11 (5.50\%) from the discharged patients. The higher positive rate of nucleic acid in the CT suspected group indicated that changes in CT imaging were more sensitive for COVID-19 diagnosis. Only 21 patients were confirmed by nucleic acid tests in all 200 symptomatic suspected cases. The nucleic acid positive rate was significant different between the CT suspected group and symptomatic suspected group (109/607, $17.96 \%$ vs $21 / 200,10.50 \%$, Chi-square value $=6.19, \mathrm{P}<0.05) .59 / 366$ close contacts were novel coronavirus positive whose rate was similar with the CT suspected group but showed no significant difference with the symptomatic suspected group $(21 / 200,10.50 \%$ vs $59 / 366,16.12 \%$, Chi-square value=3.37, $P=0.06)$. Notably, the discharged patients could be nucleic acid positive again even their previous tests were negative when discharged from hospitals. 11/59 (18.64\%) discharged patients were re-hospitalized for novel coronavirus positive again.

\section{Analysis by timeline}

Subsequently, we classified patients in accordance enrollment time to investigate the influence of epidemic in different stages on quarantine zones. The data we accumulate were from Feb ${ }^{\text {th }}$ (almost the peak of epidemic in Wuhan) to Mar $4^{\text {th }}$ (single digits per day). The smooth curve of daily hospitalization rate presented a declining trend (Figure 2A), indicating that the inpatient demand decreased with time. For further analysis, we termed Feb $8^{\text {th }}$ to Feb $15^{\text {th }}$ as the first week, Feb $16^{\text {th }}$ to Feb $23^{\text {th }}$ as the second week, and Feb $24^{\text {th }}$ to Mar $3^{\text {th }}$ as the third week (Table 3). The 45 cases in Mar $4^{\text {th }}$ ( 3 cases hospitalized) were excluded to calculate.

In the first week of observation, we found that 533 individuals were quarantined, of which 362 (67.92\%) were hospitalized. In the third week, the epidemic situation in Wuhan entered the transition period from the middle to the low stage. The newly diagnosed patients showed a downward trend, and the number of new suspected patients dropped to 257, hospitalized with 47 (18.29\%). Through statistical analyzing, 397 were quarantined in the second week, finally 127 (31.99\%) were hospitalized. A. Overall data trends suggest a decline in the number of patients in isolation per week while a same trend in the proportion of patients admitted. The chi-square test approved that the difference was significant $(67.92 \%$ vs $31.99 \%$ and $18.29 \%, P<0.05$; Figure 2B). Besides, according to the classification statistics (Table 3 ), in the first week, a total of 327 suspected patients, 33 symptomatic patients, 153 close contacts, and 20 discharged patients were received; afterwards 117 suspected cases, 129 symptomatic cases, 120 close contacts and 31 discharged cases were received in the second week. To the third week, which includes 125 suspected patients 38 symptomatic patients, 86 close contacts, 8 discharged patients. In addition, for the number of nucleic acid positive cases, $144(72.00 \%)$ were in first week, 39 (19.50\%) cases in the second week, and $17(8.50 \%)$ cases in the third week. The proportions and ratios decreased, and the chi-square test showed significant differences $(P<0.05)$. Besides, the rate of nucleic acid positive in close contacts group also decreased with time $(104 / 153,67.97 \%$ vs $42 / 12035.00 \%$ vs $13 / 8615.11 \%, P<0.05$; Figure $2 C)$. 


\section{Discussion}

In the course of the outbreak of COVID-19 in Wuhan, the infection population base was unknown and medical resources were extremely exhausted. In order to cut off the transmission and set up the preclinical triage steps, local authorities had set up hotels as quarantine zones with medical personnel. The field data provided in this manuscript intensely highlighted the significance of quarantine zones in the process of epidemic control. Briefly, in 22 days, the 14 quarantine zones in the Qiaokou District have confirmed 200 patients with COVID-19 nucleic acid positive; 540 cases were adequately sent to hospital for further treatment, 607 cases were de-isolated from quarantine and 59 patients were accepted for recovery. Except for cases confirmed COVID-19 positive after being sent to the hospital, the 200 cases confirmed at quarantine zones could have contracted 618-692 people with the virus if not properly isolated, using the basic infection index and R0 described by literature (3.09 3.46) ${ }^{4,5}$. Therefore, quarantine zones played a pivotal role in isolation, triage, observation and rehabilitation.

According to statistics, there are 136 communities in the Qiaokou District of Wuhan, 58 of which were sampled in this work, covering a large number of communities and a wide range of regions. In terms of population and spatial distribution, sampling survey samples were evenly distributed in each community of Qiaokou District, Wuhan, reflecting the randomness of the samples. Thus, data in quarantine zones were a unique perspective to observe the epidemic situation. The total number of patients in the zone directly reflected the trend of the overall epidemic. Statistics results found, although female constitute the main part of the isolated population, they displayed a significant lower rate of hospitalization than male, which was in accordance with the report that male seemed to be more susceptible of COVID-19 ${ }^{6}$. Besides, age contribution demonstrated that populations within 41-70y were the highest proportion of isolation and hospitalization ${ }^{7}$. The epidemic of COVID-19 in Wuhan came to its peak at the middle of February and decreased since then. The same trend was observed for the total number of cases per week and the number of cases who needed to be hospitalized in the quarantine zones.

Based on the epidemiological history and clinical manifestations of the people who came to the quarantine zones, we artificially divided the entire population into 4 categories: CT suspected, symptomatic suspected, close contacts and discharged patients. The majority of suspected cases had CT scan showing possible viral pneumonia before coming to quarantine zones ${ }^{8}$. Nevertheless, due to the limited supply of nucleic acid test kits at the early stage of epidemic out break and sensitivity issue affected by sampling, these CT suspected cases could not be confirmed as COVID-19 patients at that time. However, approximately half of the CT suspected cases were admitted to the hospitals and eventually confirmed as COVID-19 positive before isolation end. Hence CT scan seems to be a powerful screening tool for circumstances that nucleic acid test kits are in shortage or unavailable ${ }^{9}$. Moreover, as a mean of assessing the condition at the quarantine zones, CT scan was also important. One standard for de-isolation was that the effusion on CT image should be significantly absorbed and improved. Actually, half of the cases, whose lesions in CT clearly absorbed during their stay in quarantine zones, suggesting a possibility of self-healing of the disease, which was also related to the self-limitation of the 
virus. On the other hand, some cases suffered obvious progression in $\mathrm{CT}$, which was one of the important indications for hospitalization. The differences in the degree of pulmonary infection, basic status, immune function, and even genetic background between self-healers and those who progressed in the quarantine zones were extremely interesting topics, which deserves further investigation.

During the peak of the virus outbreak in Wuhan, the incidence of respiratory diseases such as influenza and chronic obstructive pulmonary disease was also high due to the cold weather, which confronted the medical system with challenges on the differential diagnosis of novel coronary pneumonia. It has been reported that the main symptoms were fever (98\%), cough (76\%), myalgia or fatigue (44\%), dyspnea (55\%), and lymphopenia (63\%). And atypical symptoms included sputum (28\%), headache (8\%), hemoptysis $(5 \%)$, and diarrhea $(3 \%)^{10}$. Generally, any patients with respiratory symptoms could have been considered as suspected cases. However, through the medical observation at quarantine zones, we found that part of these cases presented purely other respiratory diseases, and they could be released from quarantine zones after 14 days of isolation and a series of checks mentioned above. Further analysis demonstrated that the rate of hospitalization and positive COVID-19 nucleic acid in symptomatic suspected group were both lower than those of CT suspected cases. As a result, we separate the symptomatic group from the suspected patients for analysis. It was also important to point out that CT scan, but not symptomatic, seemed to be sufficient factor for suspected infection of novel coronavirus2019 , as well as the evidence for de-isolation.

Discharged patients also need isolation as the infection of COVID-19 was characterized as insidious, and the course of the disease was prone to prolong. Isolation in the quarantine zone for at least 14 days greatly reduced the possibility of continued infection caused by re-infection after discharged from the hospital. In the early stage of outbreak, the medical resources in Wuhan were extremely scarce, so the early discharge standard was inflammation of the lungs showed better absorption in CT and respiratory nucleic acid was negative for two consecutive times (one-day sampling time interval at least). Our data suggested that part of patients might switch into nucleic acid-positive again after discharged, which was probably because of prolonged disease. Therefore, in the case of sufficient medical resource in the middle and late stages of the epidemic, the discharge standards turned stricter, which includes serological testing as a basis to further reduce the possibility of re-admission. And the positive rate of nucleic acid hospitalized from discharged patients significantly decreased after that. This experience could be helpful to other countries as an important reference.

Close contacts were an important factor in the management of infectious diseases outside hospital ${ }^{11}$. Since the population might generally be susceptible to the COVID-19, close contacts directly determine the number of infections. Isolation in quarantine zones could cut off the transmission and stratify highrisk cases who would progress. Benefit from early discovery and efficient isolation, we found that close contact cases, the number of patients who had to be hospitalized due to progression in CT scan or worsening symptoms gradually decreased, meaning fewer patients admitted and epidemic situation relieved. There might be two reasons for this decline: undoubtedly, one was for the strict control measures in Wuhan that had significantly reduced the spread of the virus. The second, the virus was likely to 
mutate in the population, with the decrease of infectivity and pathogenicity gradually ${ }^{12}$. Another possible evidence for the less infectivity was the rate of nucleic acid positive in close contacts group significantly decreased. Nevertheless, the overall hospitalization rate in close contacts group was over $40 \%$, suggesting that COVID-19 was highly contagious that any personnel who may have the opportunity to be in close contact with confirmed cases should properly and cautiously take protective measures.

In summary, we describe the triage strategy and process of 1232 cases at quarantine zones from Qiaokou District, Wuhan. The quarantine procedures were helpful and played a pivotal role in identification of cardinal number, cutting off the community transmission, reducing the scope of screening, relieving medical pressure and rehabilitation therapy. This protocol adopted in Wuhan provided countries around the world with valuable experience in controlling the COVID-19.

\section{Declarations}

\section{Acknowledgements}

The authors thank all of the doctors, nurses, staff and patients who have contributed to this work in all quarantine zones.

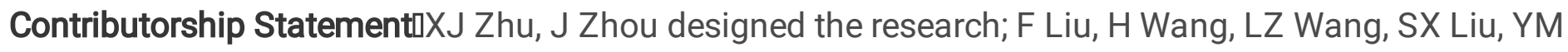
Zhang, WL Fu collected the information; YX Zhang, J Wang and XJ Zhu analyzed results and made the Figures and tables; XJ Zhu, J Wang, YT Tang, S Zhou and F Liu wrote the paper; J Wang, XJ Zhu, J Zhou and $\mathrm{H}$ Wang interpreted data and reviewed the paper.

\section{Funding}

There is no funding in study design, data collection, data analysis, data interpretation, or writing of the report.

\section{Competing interests}

The authors declare that they have NO conflict of interest.

\section{Patient consent for publication}

Not required.

\section{Ethics approval}

The research protocol was assessed and approved by the Ethical Committee of the Tongji Hospital, Tongji medical college, Huazhong University of Science and Technology.

\section{Data availability statement}


No additional data available.

\section{References}

1. Zhu N, Zhang D, Wang W, et al. A Novel Coronavirus from Patients with Pneumonia in China, 2019. N Engl J Med 2020;382:727-33.

2. Rothe $C$, Schunk M, Sothmann P, et al. Transmission of 2019-nCoV Infection from an Asymptomatic Contact in Germany. N Engl J Med 2020;382:970-1.

3. Phan LT, Nguyen TV, Luong QC, et al. Importation and Human-to-Human Transmission of a Novel Coronavirus in Vietnam. N Engl J Med 2020;382:872-4.

4. Chan JF, Yuan S, Kok KH, et al. A familial cluster of pneumonia associated with the 2019 novel coronavirus indicating person-to-person transmission: a study of a family cluster. Lancet 2020;395:514-23.

5. Li Q, Guan X, Wu P, et al. Early Transmission Dynamics in Wuhan, China, of Novel CoronavirusInfected Pneumonia. N Engl J Med 2020.

6. Lu R, Zhao X, Li J, et al. Genomic characterisation and epidemiology of 2019 novel coronavirus: implications for virus origins and receptor binding. Lancet 2020;395:565-74.

7. Chen N, Zhou M, Dong X, et al. Epidemiological and clinical characteristics of 99 cases of 2019 novel coronavirus pneumonia in Wuhan, China: a descriptive study. Lancet 2020;395:507-13.

8. Salehi S, Abedi A, Balakrishnan S, Gholamrezanezhad A. Coronavirus Disease 2019 (COVID-19): A Systematic Review of Imaging Findings in 919 Patients. AJR Am J Roentgenol 2020:1-7.

9. Nakajima K, Kato H, Yamashiro T, et al. COVID-19 pneumonia: infection control protocol inside computed tomography suites. Jpn J Radiol 2020.

10. Chen H, Guo J, Wang C, et al. Clinical characteristics and intrauterine vertical transmission potential of COVID-19 infection in nine pregnant women: a retrospective review of medical records. Lancet 2020;395:809-15.

11. Chinazzi M, Davis JT, Ajelli M, et al. The effect of travel restrictions on the spread of the 2019 novel coronavirus (COVID-19) outbreak. Science 2020.

12. Huang C, Wang Y, Li X, et al. Clinical features of patients infected with 2019 novel coronavirus in Wuhan, China. Lancet 2020;395:497-506.

\section{Tables}

Table 1. Demographic and classification of all cases. 


\begin{tabular}{ll} 
& Total $(\mathbf{n}=\mathbf{1 2 3 2})$ \\
\hline Male: Female & $577: 655$ \\
\hline Median age (range), Year & $52(2-93)$ \\
\hline SubgroupØn (\%) & \\
\hline CT Suspected & $607(49.27)$ \\
\hline Symptomatic Suspected & $200(16.23)$ \\
\hline Close contacts & $366(29.71)$ \\
\hline Discharged patients & $59(4.79)$
\end{tabular}

Table 2. Redistribution of the cases and their reason 


\begin{tabular}{|c|c|c|c|}
\hline & Hospitalized & $\begin{array}{l}\text { Isolation } \\
\text { Terminated }\end{array}$ & $\begin{array}{l}\text { Isolation } \\
\text { Continued }\end{array}$ \\
\hline Total $1232, \mathrm{~N}(\%)$ & $540(43.83)$ & $603(48.94)$ & $89(7.22)$ \\
\hline CT Suspected, n (\%) & $306(50.41)$ & $272(44.81)$ & $29(4.78)$ \\
\hline Nucleic acid test positive, $\mathrm{n}(\%)$ & $109(35.62)$ & & \\
\hline Progression of CT, n(\%) & $94(30.72)$ & & \\
\hline Aggravation of symptoms, n(\%) & $50(16.34)$ & & \\
\hline Other reason, n(\%) & $53(17.32)$ & & \\
\hline Symptomatic Suspected, n (\%) & $53(26.50)$ & $114(57.00)$ & $33(16.50)$ \\
\hline Nucleic acid test positive, n(\%) & $21(39.62)$ & & \\
\hline Progression of CT, n(\%) & $5(9.43)$ & & \\
\hline Aggravation of symptoms, n(\%) & 18(33.96) & & \\
\hline Other reason, n(\%) & $9(16.98)$ & & \\
\hline Close contacts, n (\%) & $164(44.81)$ & $190(51.91)$ & $12(3.28)$ \\
\hline Nucleic acid test positive, n(\%) & $59(35.98)$ & & \\
\hline Progression of CT, n(\%) & $22(13.41)$ & & \\
\hline Aggravation of symptoms, n(\%) & $38(23.17)$ & & \\
\hline Other reason, n(\%) & $45(27.44)$ & & \\
\hline Discharged patients, n (\%) & $17(28.81)$ & $27(45.76)$ & $15(25.43)$ \\
\hline Nucleic acid test positive, n(\%) & $11(64.71)$ & & \\
\hline Progression of CT, n(\%) & $3(17.65)$ & & \\
\hline Aggravation of symptoms, n(\%) & $1(5.88)$ & & \\
\hline Other reason, n(\%) & $2(11.76)$ & & \\
\hline
\end{tabular}

Table 3. Distribution of cases over time 
Number (hospitalized) First week Second week Third week Total

\begin{tabular}{lllll}
\hline CT Suspected & $327(220)$ & $117(56)$ & $125(29)$ & $569(305)$ \\
\hline Symptomatic Suspected & $33(25)$ & $129(25)$ & $38(6)$ & $200(56)$ \\
\hline Close contacts & $153(104)$ & $120(42)$ & $86(13)$ & $359(159)$ \\
\hline Discharged patients & $20(13)$ & $31(4)$ & $8(0)$ & $59(17)$ \\
\hline Total & $533(362)$ & $397(127)$ & $257(48)$ & $1187(537)$
\end{tabular}

\section{Figures}

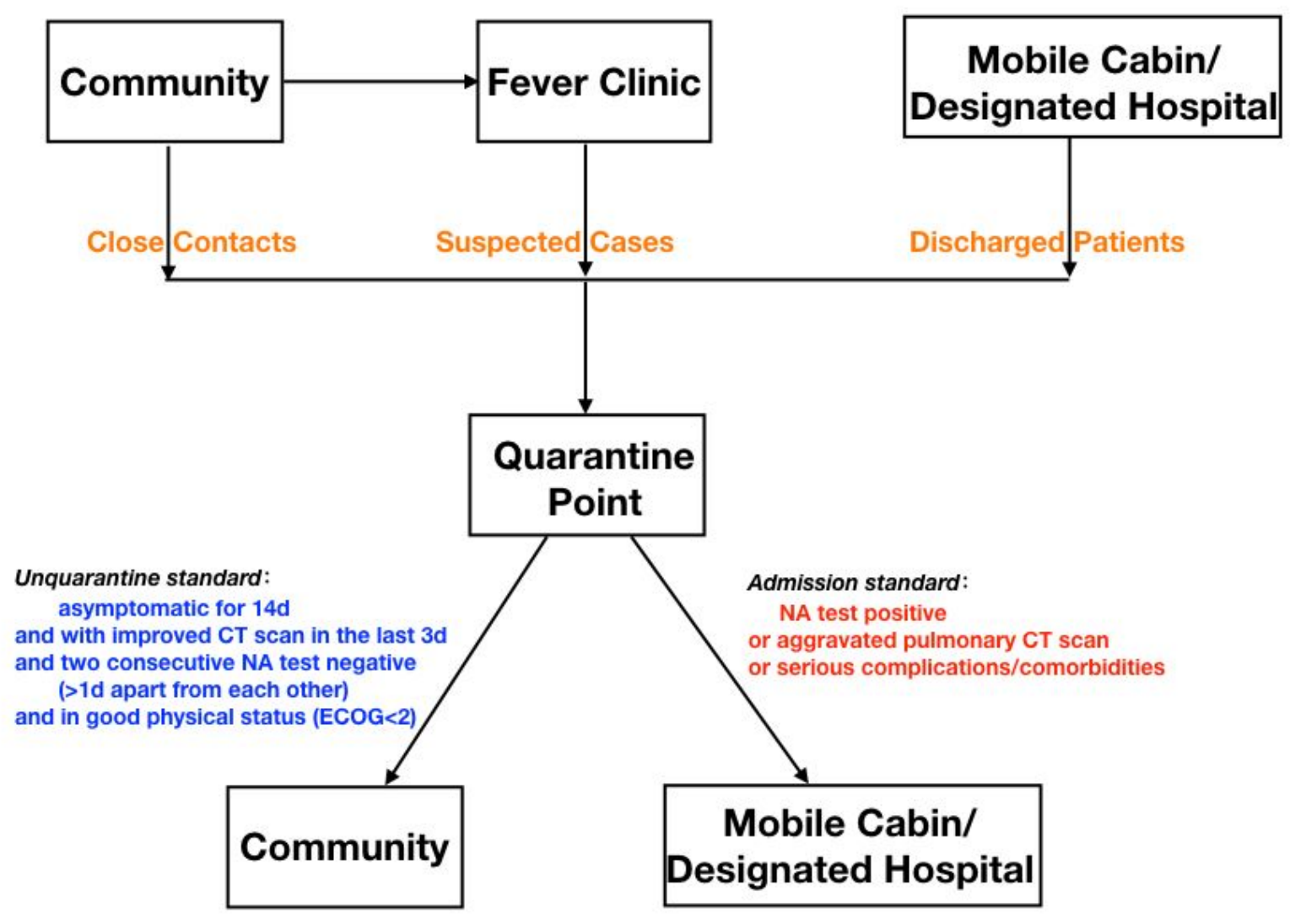

Figure 1

Protocol of quarantine zones in Qiaokou District. When the patients have suspected pneumonia symptoms, they came to the Fever Clinic and accept nucleic acid and CT examination. Suspected cases were those who have imaging features of viral pneumonia, PCR negative and symptomatic or 
asymptomatic. People with fever or respiratory symptoms were termed as symptomatic cases (PCR negative and without viral pneumonia changes in $\mathrm{CT}$ ). The two groups came to quarantine zones from Fever Clinic. Those who have history of close contact with confirmed cases in the absence of effective protection came to quarantine zones from community. Patients discharge from the designated hospital also need to isolate in the quarantine zones. According to the unquarantine and hospitalized standard, these cases were eventually released from quarantine zone or sent to hospital for further medical care.

A
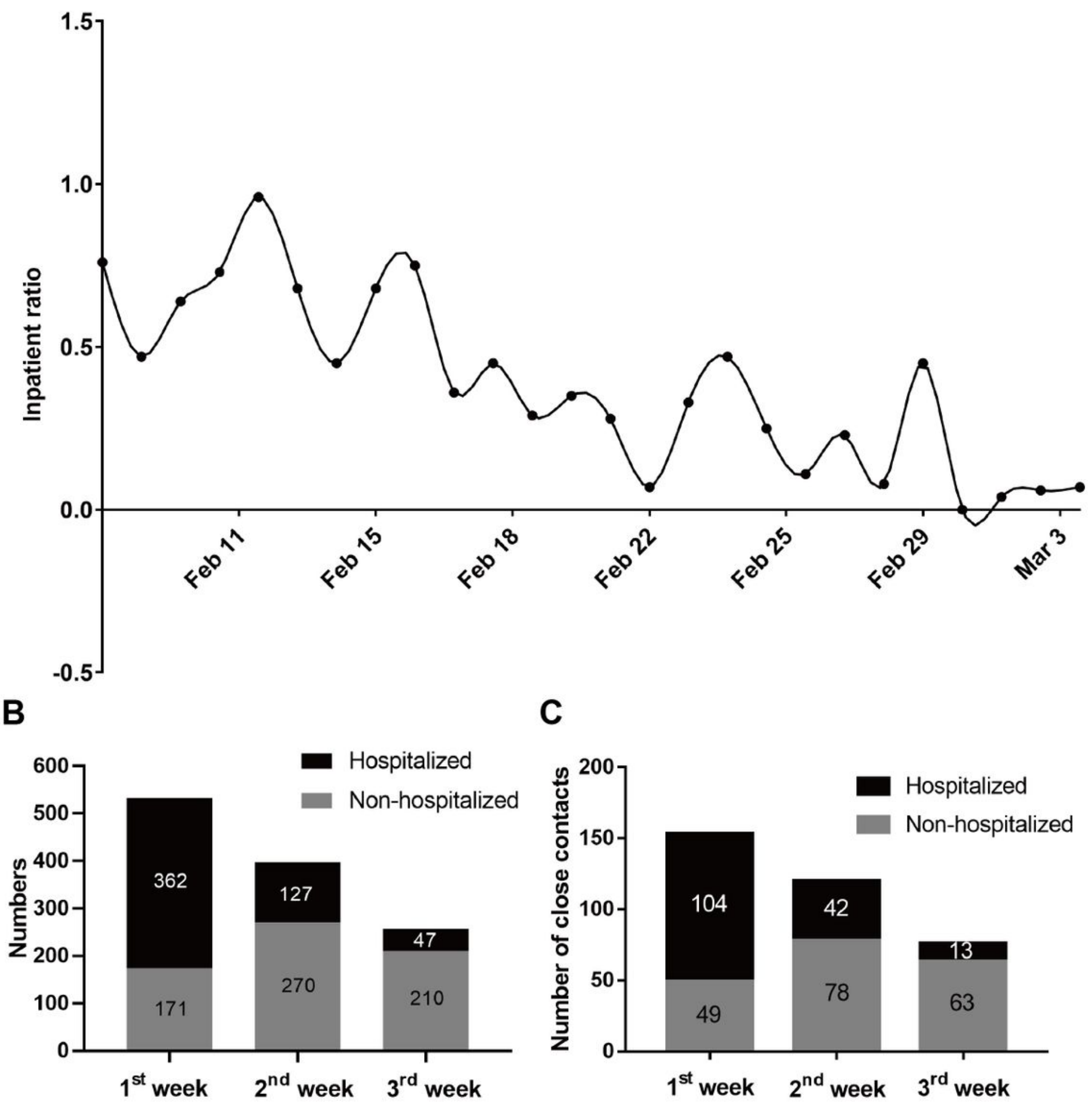

Figure 2 
Distribution of cases by timeline. A. Smooth curve of daily hospitalization rate. We termed Feb 8th to Feb 15th as the first week, Feb 16th to Feb 23th as the second week, and Feb 24th to Mar 3th as the third week. B. 362/533 (67.92\%) individuals were hospitalized the first week, 127/397 in the second week and $47 / 257(18.29 \%)$ in the third week. The chi-square test approved that the difference was significant ( $362 / 533$ vs $127 / 397$ and $47 / 257, P<0.05$ ). C. The rate of nucleic acid positive in close contacts group also decreased with time $(104 / 153$ vs $42 / 120$ and $13 / 86, P<0.05)$. 